\title{
Heme overdrive rewires pan-cancer cell metabolism
}

Swamy R. Adapa ${ }^{1,7^{*}}$, Gregory A. Hunter ${ }^{2 *}$, Narmin E. Amin ${ }^{3}$, Christopher Marinescu ${ }^{1}$, Andrew Borsky ${ }^{2}$, Elizabeth M. Sagatys ${ }^{4}$, Said M. Sebti ${ }^{5}$, Gary W. Reuther ${ }^{3}$, Gloria C. Ferreira $^{2,6,7}$, Rays H.Y. Jiang ${ }^{1,7 \ddagger}$.

1 USF Genomics Program, Center for Global Health and Infectious Diseases, College of Public Health, University of South Florida, Tampa, FL 33612, USA.

${ }^{2}$ Department of Molecular Medicine, Morsani College of Medicine, University of South Florida, Tampa, FL 33612, USA.

${ }^{3}$ Department of Molecular Oncology, H. Lee Moffitt Cancer Center and Research Institute, Tampa, FL 33612, USA.

${ }^{4}$ Department of Pathology, H. Lee Moffitt Cancer Center and Research Institute, Tampa, FL 33612, USA.

${ }^{5}$ Department of Pharmacology \& Toxicology, Massey Cancer Center, Virginia Commonwealth University, Richmond, VA 23298, USA

${ }^{6}$ Department Department of Chemistry, College of Arts and Sciences, University of South Florida, Tampa, FL 33620, USA.

${ }^{7}$ Global and Planetary Health, College of Public Health, University of South Florida, Tampa, FL 33612, USA.

*These authors contributed equally to the work ‡Corresponding author: jiang2@usf.edu

Keywords: Heme, iron, cancer, Warburg effect, ferroptosis, apoptosis, porphyrin, cancer metabolism, single-cell RNAseq 


\title{
Heme overdrive rewires pan-cancer cell metabolism
}

\begin{abstract}
: (125 words)
All cancers reprogram their metabolism. In this study, we report that all cancer cells employ imbalanced and dynamic heme metabolic pathways essential for their oncogenic growth and coined the term 'heme overdrive'. Three key characteristics define heme overdrive, i.e., cancer universality, cancer essentiality and cancer specificity. While heme overdrive is absent in diverse differentiated cells or somatic stem cells, it is present in hundreds of cancers, and is delineated in patient-derived tumor progenitor cells by single cell RNAseq. The only exception to heme overdrive in normal tissues is identified in preimplantation human embryos, where heme overdrive likely links to embryonic omnipotency. Among the major drivers are proteins involved in biosynthesis of heme intermediates (hydroxymethylbilane synthase (HMBS) and uroporphyrinogen decarboxylase (UROD)) and heme trafficking (FLVCR1). CRISPR/Cas9 editing to engineer leukemia cells with impaired heme biosynthesis steps confirmed our whole genomic data analyses that heme overdrive is linked to oncogenic states and cellular differentiation. Finally, we devised a novel "baitand-kill" strategy to target this cancer metabolic vulnerability.
\end{abstract}

One-Sentence Summary: (125 characters and spaces)

Heme overdrive re-programs cellular metabolism across diverse types of cancer. 
Main Text $(3,000$ to 10,000 words $)$

\section{Introduction}

Heme biosynthesis is one of the most efficient metabolic pathways in humans. A total of 270 million molecules of heme are produced for every red blood cell (RBC) ${ }^{1}$, at a rate of over 2 million RBCs per second ${ }^{2}$. Heme, a porphyrin ring encaging most of the iron in humans, functions as an essential prosthetic group of numerous proteins with roles ranging from signal sensing, DNA binding, microRNA splicing and processing to enzymatic catalysis ${ }^{3-21}$. To ensure the enormous output of heme biosynthesis, the supply of substrates, intermediates and end-products in the pathway is tightly regulated and precisely balanced 7,22 . Heme is a double-edged sword for cell growth; it is essential in the "right amount" 23 and if not can be toxic ${ }^{24,25}$ via unique forms of cell death.

Cancer cells reprogram metabolic pathways to fuel anabolic growth, rapid propagation, and efficient nutrient acquisition ${ }^{26-28}$. Although some studies have implicated heme in carcinogenesis through cytotoxic heme-derived compounds ${ }^{29,30}$, lipid peroxidation ${ }^{31}$, oxidative damage ${ }^{32}$, intestinal flora toxicity ${ }^{33}$ and energy production ${ }^{34}$, little is known about how heme biosynthesis deregulation and heme trafficking alterations contribute to tumor dependence on heme for survival. Here we describe 'heme overdrive', a novel cancer cell metabolic reprogramming characterized by imbalanced and dynamic heme metabolic pathways essential for cancer cell growth.

"The Warburg effect" 26,35 and "glutamine addiction" 36,37 are two altered forms of metabolism found in cancer cells. Therapeutic intervention based on these altered cancer metabolic pathways are challenging because glycolysis and glutamine metabolism are required in every cell and thus such therapeutic approaches lead to non-specific toxicity. By contrast, heme overdrive is an ideal cancer metabolic pathway for therapeutic targeting as we demonstrate that it is: 1 ) cancer specific (i.e., it is absent in normal cells), 2) universal (i.e., it is present in all cancers), and 3) cancer cell essential (i.e., cancer cells are addicted to it).

\section{Results}

\section{Cancer is characterized by aberrant heme metabolism}

Compared to normal cells, cancer cells have enhanced metabolic dependencies ${ }^{26-28}$. Our initial data analyses of genome-scale CRISPR/Cas9-gene loss-of-function from the publicly available project DepMap screens of cell lines derived from metastatic cancers 38 indicated that cancer cells depend on heme synthesis (fig. S1) (Supplemental Table S1). Significantly, these metastatic cancers developed dependencies on heme metabolism-related proteins, such as uroporphyrinogen III decarboxylase (UROD), the enzyme that catalyzes the fifth step in the heme biosynthetic pathway. These cancers also depend on a set of hemoproteins, such as cytochrome c-1 (CYC1), succinate dehydrogenase complex subunit $\mathrm{C}$ (SDHC) for cellular respiration, and DGCR8 for microRNA biogenesis ${ }^{39}$, each of which uses heme as a co-factor. Unexpectedly, the $F E C H$ gene that encodes ferrochelatase that catalyzes the final critical step of heme biosynthesis is dispensable in several cancers, suggesting that cancer cell lines are 
capable of bypassing endogenous biosynthesis of heme in vitro, yet are still dependent on genes encoding enzymes that mediate intermediate steps of heme biosynthesis. This initial analysis suggests that cancer is dependent on imbalanced heme metabolism (Fig. $1 \mathrm{~A})$.

Next, we investigated the genes that encode proteins acting as gatekeepers for heme biosynthesis. The first committed and key regulatory step in mammalian heme biosynthesis is catalyzed by 5-aminolevulinic acid (ALA) synthase (ALAS; EC 2.3.1.37) 22,40. Two chromosomally distinct genes, ALAS1 and ALAS2, encode the housekeeping and erythroid-specific ALAS isoforms, respectively ${ }^{41}$. While ALAS1 is expressed in every cell, the expression of $A L A S 2$ is restricted to developing erythrocytes ${ }^{40}$. We examined the data generated from genome-wide CRISPR-based screens of diverse cancer cell lines, which were designed to provide a complete gene essentiality data set for these cell lines ${ }^{38,42}$. In contrast to $A L A S 2$, essentiality of $A L A S 1$ is a feature of diverse cancer cells independent of cancer type (fig. S2A). Consistent with these forward genetics results, the gene expression patterns in $\sim 10,000$ patient tumors from the Genotype-Tissue Expression (GTEx) ${ }^{43,44}$ and The Cancer Genome Atlas (TCGA) ${ }^{45}$ datasets show that ALAS2 expression is absent in most tumors except myeloid leukemias (fig. S2B). These results show that, despite ALAS2 being responsible for the majority of heme production $(\sim 85 \%)$ in humans, many cancer cells, including erythroleukemia of red blood cell lineages (e.g. HEL), require ALAS1.

\section{Heme overdrive operates in diverse cancers and early embryos}

To better understand the molecular and genetic mechanisms underlying the cancer dependency on heme metabolism, we determined gene essentiality from genome-scale CRISPR/Cas9 loss-of-function screens in over 300 human cancer cell lines covering different cell lineages and estimated gene dependency 38,42 . Then, we focused on the gene dependencies associated with the eight enzymatic steps of the heme biosynthetic pathway by computing the respective pan-cancer essentiality scores, similar to the lethality scores used in the development of a cancer dependency map (DepMap) (42, 43). Gene essentiality was estimated from gene $X$ dependency inferred from CRISPR/Cas9 gRNA gene $X$ knockout. Lower score values indicate larger effect of gene $X$ loss on cell viability, and hence cell lines with lowest and highest scores are most and least gene X-dependent, respectively. Consistent with recent cross-platform studies ${ }^{46,47}$, over 2000 genes were defined as pan-cancer essential, based on the distribution of their essentiality scores in over 300 cancer lines derived from 27 of the most prevalent cancer types. The gene essentiality scores were confirmed by using the same method on the total DepMap collection (DepMap release 21Q3) of over 1000 cell lines (Pearson's R > $0.9, \mathrm{p}<0.001)$. Strikingly, instead of finding the expected complete and balanced heme biosynthetic pathway, partial heme biosynthetic pathways were found in hundreds of different types of cancers (fig. S3) (Supplemental Table S2 and S3). Specifically, as shown in Fig. 1B and fig. 3S, the pan-cancer gene essentiality is the highest for the genes encoding the enzymes responsible for the fifth and sixth steps of the pathway, UROD and coproporphyrinogen III oxidase (CPOX), respectively. This result was notably surprising, as the genes for the ALAS isoforms, which catalyze the initial and rate-limiting step in the pathway, and the gene for $\mathrm{FECH}$, the final enzyme in the pathway that 
catalyzes heme production, had lower essentiality values and were dispensable in several cancers (Fig. 1B). Though the heme biosynthesis genetic dependences of 27 major forms of human cancer vary, the estimated patterns of essentiality across the cancer lines clearly revealed a range of heme and heme precursor requirements. These results uncovered the "imbalanced" nature of heme biosynthesis in cancer, with many cancers surviving without functional first and terminal enzymatic steps, but dependent on the intermediate steps (fig. S3 and fig. S4). The gene essentiality analysis also highlighted the importance of heme trafficking (e.g., heme importer FLVCR2) and key hemoproteins (e.g., CYC1) in cancer cells survival (Fig. 1B and fig. S3).

To examine cancer heme metabolism in vivo, we analyzed the in vivo mouse CRISPR/CAS9 loss-of-function and metabolic essentiality data from pancreatic and lung cancer models ${ }^{48}$. The genetic screen ${ }^{48}$ indicates that the majority of metabolic gene essentialities were similar between the in vitro and in vivo settings, with the exception of the heightened requirement of the intermediate steps of heme biosynthesis in vivo. Strikingly, out of the 2900 examined metabolic genes ${ }^{48}$, the genetic dependencies of both murine pancreatic and lung cancers were only increased for the enzymes responsible for the intermediate steps of the heme biosynthesis pathway (fig. S5) (Supplemental Table S4). The essentiality extent of either $A L A S$ or FECH, which code for the first and terminal enzymes of the heme biosynthesis pathway, respectively, did not differ significantly between the in vivo murine cancer models. This analysis of the CRISPR/Cas 9 loss-of-function screens performed in mouse model systems revealed that aberrant heme metabolism has increased importance in vivo for cancer cell survival (fig. S5). Furthermore, this effect is independent of tumor origin or tissue environment in pancreatic and lung cancers.

To assess heme biosynthesis in patient tumor samples, we analyzed and compared the gene expression patterns in $\sim 10,000$ tumors vs. those in normal tissues available from the GTEx project ${ }^{43,44}$ and TCGA program ${ }^{45}$. We used methods designed for pairwise comparative gene expression of GTEx and TCGA datasets ${ }^{49}$. In over $80 \%$ of all tumors, the genes for hydroxymethylbilane synthase (HMBS), the third enzyme of the heme biosynthetic pathway, and the heme exporter FLVCR1 were among the most upregulated (fig. S6A,B)(Supplemental Table S5). By the same criteria, the onco-signaling genes AURKA, KRAS and MYC were upregulated in over $90 \%, 60 \%$ and $50 \%$ of all tumor types, respectively. In contrast, expression of the genes encoding the second and terminal enzymes of the heme biosynthetic pathway, aminolevulinate dehydratase (ALAD, a.k.a. porphobilinogen synthase) and ferrochelatase (FECH), respectively, are down-regulated suggesting buildup of intermediate heme precursors in tumors. HPX, the gene for the heme-binding and scavenger hemopexin ${ }^{50}$, was also downregulated in most of the tumors (fig. S6C). This finding, which corroborates the previously reported role of hemopexin as a key player for the checkpoint in cancer growth and metastases ${ }^{51}$, leads us to suggest that heme content is high in the tumor microenvironment ${ }^{52}$. ALAS2 belongs to the $10 \%$ of genes that were not expressed in most of the large tumor collections, except myeloid leukemias ( $<2 \%$ of total tumors). Overall, our comparative analysis of the differential gene expression in tumor tissues, spanning 31 cancer types, indicated that only a few of the nine heme biosynthesis enzyme-encoding genes were upregulated in cancer cells. The unbalanced expression of the genes for heme biosynthesis and heme transporters likely elicits a lack of heme homeostasis in cancer cells, where the 
insufficient heme supply from the dedicated biosynthetic pathway is compensated with an enhanced heme flux.

Based on the gene essentiality results both in vitro (Fig. 1B, fig. S3 and fig. S4) and in vivo (fig. S5) settings, the pan-cancer gene expression patterns (fig. S6), the detected porphyrin accumulation in a wide range of tumors ${ }^{30}$, and the determined elevated heme flux in diverse cancer cells $32,34,53$, we defined the salient heme overdrive-associated features. They are: 1) heme biosynthesis enzymes with aberrantly increased (HMBS, UROS and UROD) and suppressed (ALAD and FECH) activities, 2) accumulation of heme precursors (porphyrins), and 3) enhanced heme flux (Fig. 1, A and B). This cellular status contrasts with the tightly regulated steady-state or homeostasis, defined by a flawless enzyme-catalyzed channeling of substrates to products along the heme biosynthetic pathway and circumventing the toxicity and instability of the pathway intermediates, while ensuring that the cell heme requirements are met ${ }^{7}$. With heme overdrive, the production of heme precursor molecules exceeds that of heme, porphyrin intermediates accumulate, and "imbalanced" heme biosynthesis of increased porphyrin intermediates and decreased end-product arises. Likely, to compensate for the reduced amount of heme synthesized, heme flux is increased ${ }^{30}$. Cancer cells appear to have adopted a dependence on this seemingly "inefficient" metabolic pathway designed to produce aberrant levels of heme intermediates with unknown biological functions to date.

Early embryonic stem cells represent a special metabolic state that bears similarities with that of cancer cells 54,55. Therefore, we also studied the expression of the heme biosynthetic enzyme-encoding genes in human preimplantation embryos and embryonic stem cells. Specifically, we explored a high-resolution data set generated upon single-cell RNA-sequencing in human early embryonic cells and embryonic stem cells ${ }^{56,57}$. In the human zygote and early preimplantation embryos, the level of ALAS1 expression is 100fold higher than that of $F E C H$ (Fig. 1C). This large difference in gene expression for two key enzymes in heme biosynthesis was observed in all 17 early human embryos examined (Supplemental Table S6). The large difference in expression levels is restricted to very early embryos because it rapidly narrows, via an increase in FECH expression and a decrease in ALAS1 expression after initial rounds of embryonic cell division. ALAS2 expression is not detected in any of the early human embryos, which is congruent with the non-essentiality of $A L A S 2$ in all examined cancers. As a control, the genes $A U R K A$ and SOX4 show expected expression patterns during early embryogenesis ${ }^{58,59}$. The similarity of these gene expression data with those observed in cancer cells supports the possibility that a form of heme overdrive operates in preimplantation human embryos.

\section{Heme overdrive is absent in normal cells}

To evaluate if heme overdrive is specific to cancer cells, we investigated and compared heme biosynthesis in diverse types of non-cancerous cells, i.e., normal differentiated cells, replicating fibroblasts, human primary hepatocytes, and human primary hematopoietic stem cells. We looked for evidence of heme overdrive, defined by a nonhomeostatic, imbalanced heme biosynthetic pathways and heightened accumulation of heme pathway intermediates. Using published datasets, we found that heme overdrive, observed in cancer cells, is absent in normal differentiated cells and somatic stem cells (Fig. 2A) First and consistent with this finding, normal human peripheral blood 
mononuclear cells (PBMCs) do not accumulate heme intermediates even upon ALAinduction ${ }^{60}$. Second, colon cancer cells incubated with ALA to induce protoporphyrin IX (PPIX) production do accumulate PPIX, while no significant PPIX accumulation is observed in replicating human colon-derived fibroblasts (stromal cells) previously incubated with ALA ${ }^{61}$. Further, the increased HMBS activity in colon cancer cells relative to stromal cells ${ }^{61}$ is consistent with a buildup of heme intermediates including PPIX. Third, with our previously described primary human hepatocyte system ${ }^{62}$, we demonstrate that normal, metabolically active, primary hepatocytes do not accumulate porphyrins as indicated by the absence of the PPIX fluorescence (Fig. 2B). By contrast, $>99 \%$ of liver cancer cells produced and accrued PPIX upon induction with ALA, one of the canonical features of heme overdrive.

To uncover the role of heme metabolism in normal human stem cells, we analyzed the erythropoiesis data generated by RNAi-based knockdown of gene expression in human hematopoietic progenitor cells ${ }^{63}$. By mapping normal stem cell gene essentiality following the methods described in Egan et al. ${ }^{63}$, we show that erythropoiesis is heme-dependent in both early progenitor (undifferentiated) cells and differentiated cells from normal human bone marrow-derived hematopoietic stem cells. In contrast to cancer cells, where ALAS2 is dispensable, normal human hematopoietic stem cells depend on ALAS2 for survival (Fig. 2C). The differential gene essentiality profiles between cancer and stem cells are consistent with the molecular basis for specific ex-vivo purging of cancer hematopoietic progenitor cells as a way to eradicate malignant cells that may contaminate an autologous graft and cause relapse after transplant 64,65 . Taken together, the distinct genetic essentialities and the markedly different porphyrin accumulation modes between cancer and non-cancerous cells indicate that heme overdrive is absent in somatic stem cells.

\section{CRISPR/Cas9-mediated knockout of ALAS2 and FECH validate abnormal heme metabolism underlying cell proliferation and oncogenesis}

We modified chronic myeloid leukemia K562 cells with CRISPR/Cas9-mediated knockout (KO) of the genes encoding FECH and ALAS2 (Fig. 2D). Under a heme homeostasis model, a cell line without $F E C H$ should not survive, because $F E C H$ is a single copy gene in humans and with no functional substitute ${ }^{66-68}$. Indeed, knockdown (KD) of $F E C H$ in human stem cells led to cell death ${ }^{63}$. However, under heme overdrive conditions, we predicted that cancer cells rely on heme trafficking and are addicted to the porphyrin intermediates, and thus do not depend on the final step of heme biosynthesis for survival. As hypothesized, K562 cells with the FECH edited out (K562-FECH KO) (Fig. 2D) had normal growth (Fig. 2E) under standard culture conditions. The unimpeded growth of the K562-FECH KO cells presumably indicates that at least some cancer cell lines can metabolically function as heme auxotrophs.

As predicted, K562 cells with the ALAS2 deleted (K562-ALAS2 KO) (Fig. 2D) lost their differentiation capacity although their growth was not hindered (Fig. 2E, F). Given the proposal that oxidation stress, with the involvement of reactive oxygen species (ROS), is the first step in erythroid differentiation of $\mathrm{K} 562$ cells ${ }^{69}$, we used the organic peroxide tertbutyl hydroperoxide (t-BHP) to trigger ROS-mediated erythroid differentiation. As early as 30 minutes following t-BHP-mediated ROS induction, the parental K562 cells started synthesizing heme, an early step in cellular differentiation ${ }^{69}$, while the K562-ALAS2 KO 
cells failed to initiate this metabolic step, indicated by the lack of heme production (Fig. 2F). ${ }^{69}$. Further, at 48 hours, the parental K562 cells readily differentiated into erythroid cells, but the K562-ALAS2 KO cells continued to proliferate and failed to differentiate, as expected because ALAS2 is required for erythroid differentiation. Conceivably, in the absence of endogenous production of heme destined to hemoglobin, the erythroid lineage commitment is blocked, forcing the K562-ALAS2 KO cells to an obligatory heme overdrive path and permanent arrest in a non-differentiable/proliferation or cancerous state.

\section{Single-cell transcriptomics reveal heme overdrive in AML patient biopsies}

To study heme overdrive in primary cancer cells, we performed single-cell RNAseq on biopsies from acute myeloid leukemia (AML) patients (Supplemental Table S7 and S8). For each patient, we obtained around 2000 single-cell transcriptomes, and we analyzed cells of various differentiation stages and cell types. Interestingly, in 2 patients, only a minority of cells (5-10\% of total cells) were identified as cancer progenitors with hyper proliferation features (Fig. 3A), and they exhibited hybrid features of mature erythroid cells (e.g. expressing the genes $H B B$ and GYPA for hemoglobin $\beta$ and glycophorin $A$, respectively) and stem cells (e.g. expressing SOX4). The cancer progenitor cells (Fig. 3B) had both mature cell features, e.g. HBB expression, and early progenitor properties, e.g. proliferative phenotype. Specifically, they were characterized by elevated iron and heme metabolic gene expression, cell proliferation markers, KRAS and anabolism process genes, yet they had reduced normal stem cell progenitor marker gene expression e.g., SOX4 and JUN (Fig. 3C) ${ }^{70,71}$. Additionally, cancer/AML progenitor cells exhibited imbalanced heme biosynthesis, exemplified by elevated gene expression for enzymes that catalyze the intermediate steps of heme biosynthesis, e.g. HMBS and UROD (Fig. $3 \mathrm{D}$ and $\mathrm{E}$ ). The cancer progenitor cells also had a higher expression level of genes related to heme trafficking such as FLVCR1 and DNM2 (Fig. 3C). Our results show that these cancer progenitor cells exhibit features of heme overdrive, consistent with the abnormal heme anabolism and endogenous PPIX accumulation upon ALA administration in leukemia cells compared to normal PBMCs ${ }^{60}$. Notably, in two solid tumors (Fig. 3F) with independently generated single cell transcriptomes ${ }^{72,73}$, the genes encoding the enzymes for the intermediate steps of heme biosynthesis were similarly overexpressed in the cancer progenitor cells (Fig. 3F).

From our single cell transcriptome results, we searched for clues of specialized oncogenic microenvironments that support enhanced substrate trafficking and molecular interactions. Interestingly, only the cancer progenitor cells highly express cell-to-cell interaction-related genes, e.g., ICAM4, MAEA, ITGA4 (fig. S7A), which are critical in establishing the stem cell niche for erythropoiesis ${ }^{74,75}$. The specific expression of these niche interaction genes suggest that the cancer progenitor cells exist in a specialized niche with intimate contact with neighboring cells and extracellular matrix.

Expression was remarkably enhanced for genes encoding proteins involved in metabolic substrate trafficking processes in the cancer progenitor cells, including genes for key protein players in lipid import, purine and pyrimidine salvage pathways (fig. S7B). These specific metabolic flux related genes, such as DNM2 (endocytosis), APOC1 (lipid transport), MRI1 (L-methionine salvage), MTAP (NAD salvage), and SLC29A1 
(nucleoside import) indicate that the cancer progenitor cells represent hubs of metabolic trafficking activities. In particular, the lipid import gene APOC1 is expressed in cancer progenitor cells, but not in the other cell populations from the same patient samples (Fig. S8, A and B). The metastatin S100 family members linked to tumor niche construction ${ }^{76}$ are also enriched in the progenitors. These potential dynamic metabolic trafficking patterns inferred from the abundant expression of metabolites import genes in cancer early progenitors (Fig. S7C) are reminiscent of 'metabolic parasitism' 77 , a bioenergetically favorable process ${ }^{78}$ that utilizes energy-rich and pre-existing macromolecules to fuel robust cell proliferation.

\section{Targeting cancer heme overdrive with a bait-and-kill strategy}

Two aspects of cancer heme overdrive offer opportunities for therapeutic intervention. First, an imbalanced heme biosynthetic pathway (i.e., with aberrantly increased and suppressed enzyme activities) is absent in normal cells. Second, heme overdrive is inducible hundreds to thousands-fold in cancer cells, but not in non-cancerous cells. In fact, photodynamic therapy (PDT), which uses ALA to induce biosynthesis and accumulation of photosensitizing porphyrins, exemplifying the inducible principle of heme overdrive, has been used for imaging and treatment for decades ${ }^{79-81}$. Thus, heme overdrive may be a unique metabolic reprogramming feature of cancer cells that can be therapeutically exploited. Since an imbalanced heme flux is fundamental to heme overdrive, cancer cells may be made particularly vulnerable to PPIX-induced cytotoxicity. Selective cancer cell death could possibly be achieved by activating PPIX-induced cytotoxicity with a two-step "bait-and-kill" strategy.

Knowing that cancer cells readily take up the heme precursor ALA and bypass the first and rate-limiting step of porphyrin biosynthesis catalyzed by ALAS, we set to assess our engineered two-step process for cancer metabolic death. This two-step process involves first, to 'bait' with ALA to induce PPIX accumulation up to a few hundred-to-thousand-fold higher concentration as compared to control cells (Fig. 4A and B), and second, to 'kill' with a compound that exploits the metabolic stress associated with porphyrin accumulation.

To exploit the metabolic stress (e.g., lipid peroxidation) associated with porphyrin accumulation, we treated the human erythroleukemia HEL cell line with a range of concentrations of RSL3, an inhibitor of the antioxidant enzyme glutathione peroxidase 4 (GPX4), a phospholipid hydroperoxidase that protects cells against membrane lipid peroxidation. As such, inhibition of GPX4 with RSL3 induces ferroptosis, an irondependent form of programmed cell death highly relevant to iron and heme metabolism 82. Pretreatment of HEL cells with ALA increased RSL3 sensitivity at least 1000 -fold (Fig. 4, $C$ and D). In contrast, ALA pretreatment did not sensitize cells to ganetespib, a heat shock protein inhibitor with antineoplastic activity ${ }^{83}$ suggesting that ALA is not a general inducer of cell death. 


\section{Discussion}

For over a century, the association between photosensitizing porphyrins and cancer has been recognized ${ }^{84}$, and the preferential porphyrin accumulation in cancer cells has been used in the development of light-based therapies as early as $1911^{85}$. Our results show that heme overdrive, characterized by an imbalanced and dynamic porphyrin production, is ubiquitous to cancer cells and provide the likely cellular basis for specific light-based therapies. With a new understanding of this aberrant metabolic pathway in cancer, heme overdrive may offer novel vulnerabilities and opportunities for cancer therapeutic intervention.

Cancer metabolic processes are often described in terms of aberrant gene regulation and epigenomic reconfiguration ${ }^{86-88}$. Heme overdrive likely operates through direct epigenetic regulation ${ }^{89}$ with heme/porphyrin acting as a master regulator of chromatin, such as via binding to guanine quadruplex (G4) DNA structures ${ }^{51}$. Heme, a recognized molecular sensor ${ }^{15}$, contributes - perhaps via a paracrine/autocrine mechanism, - to the regulation of genes associated with biological processes as diverse as angiogenesis, oxidative stress management, circadian rhythm adjustment ${ }^{90}$, and anabolism ${ }^{15}$. Clearly, this recognition of heme as a signaling molecule has bolstered our understanding of the multifaceted roles of heme in cancer pathogenesis ${ }^{91,92}$. Our findings provide a new line of evidence for the signaling power of heme/porphyrin, particularly in relation to cellular redox stress and growth.

Cancer cells often employ bio-energetically favorable mechanisms to fuel anabolism and growth ${ }^{78}$. For rapidly dividing cancer cells, this means a heavy reliance on utilization of salvage and scavenging ${ }^{93}$ pathways to provide structural and energetic metabolic intermediates to fuel unchecked proliferation ${ }^{78}$. With the analysis of our data on human AML single-cell transcriptomes, we found that cancer progenitor cells preferably express genes for enzymes involved in salvaging pathways for biomolecules such as NAD, purines, and pyrimidines. Interestingly, the genes SLC28A3 and SLC29A3, encoding nucleotide importers, are under direct heme epigenetic control ${ }^{89}$. Thus, to support the heavy metabolite trafficking activities, cancer heme overdrive is also probably associated with a wide range of cancer microenvironments.

Cancer cells do not exist in isolation: aggressive cancers successfully remodel not just their metabolism but also their immediate cellular environment. In fact, recently, cancerassociated stromal cells, such as cancer-associated fibroblasts (CAFs), were shown to shape the cancer cell niche and promote cancer progression ${ }^{94,95}$. Preliminary evidence suggests that heme overdrive is present in cancer niche cells, leading us to raise the intriguing possibility that targeting heme overdrive can potentially kill both the cancer and the partner cells in the tumor microenvironment (based on our analysis of available single-cell solid tumor data (unpublished results)). Such dual lethality may be attributed to the fact that CAFs are highly anabolic and have increased collagen production and growth factor secretion ${ }^{96,97}$, a set of features uniquely shared with cancer cells.

Cancer heme overdrive is similar, yet opposite in magnitude, to human porphyrias, a group of inborn genetic diseases mostly caused by reduced enzyme activities (Fig. S6A) 98. It is of interest to note that, except the erythroid lineage ALAS2 gain-of-function 
porphyria, no gain-of-function of human porphyria's (similar to cancers) have ever been reported ${ }^{98}$, suggesting possible developmental lethality. Thus, cancer's imbalanced heme biosynthesis likely causes elevated pools of heme metabolites, while most porphyria's likely cause reduced heme synthesis.

In conclusion, through whole genome CRISPR KO analysis, single-cell transcriptomics using patient-derived tumor cells, and characterization of the heme biosynthetic pathway in engineered mammalian cells, we found an unrecognized pivotal role of imbalanced and dynamic heme metabolism in oncogenesis. Heme overdrive is a process that is universal in cancer, cancer-specific and required for cancer cell survival, and may be exploitable with novel therapeutic approaches. Finally, our studies provide a basis for exploring new avenues of research on the metabolic features of cancer cells and tumor microenvironments.

\section{References}

1 D'Alessandro, A., Dzieciatkowska, M., Nemkov, T. \& Hansen, K. C. Red blood cell proteomics update: is there more to discover? Blood Transfus 15, 182-187, doi:10.2450/2017.0293-16 (2017).

2 Dean, L. in Blood Groups and Red Cell Antigens https://www.ncbi.nlm.nih.gov/books/NBK2263/ (National Center for Biotechnology Information (US), 2005).

3 Ponka, P., Koury, M. J. \& Sheftel, A. D. in Handbook of Porphyrin Science, Erythropoiesis, Heme and Applications to Biomedicine Vol. 27 (eds G. C. Ferreira, K.M. Kadish, K.M. Smith, \& R. Guilard) Ch. 129, 42-84 (World Scientific Publishing Co. Pte. Ltd., 2014).

4 Mercurio, S. et al. The heme exporter Flvcr1 regulates expansion and differentiation of committed erythroid progenitors by controlling intracellular heme accumulation. Haematologica 100, 720-729 (2015).

$5 \quad$ Kapetanaki, M. G. et al. Free heme regulates placenta growth factor through NRF2antioxidant response signaling. Free Radic Biol Med 143, 300-308, doi:10.1016/j.freeradbiomed.2019.08.009 (2019).

6 Hanna, D. A. et al. Heme dynamics and trafficking factors revealed by genetically encoded fluorescent heme sensors. Proc. Natl. Acad. Sci. (USA) 113, 7539-7544, doi:10.1073/pnas.1523802113\%J Proceedings of the National Academy of Sciences (2016).

7 Ferreira, G. C. in The Encyclopedia of Biological Chemistry Vol. 2 (ed W. and Lane Lennarz, M. D.) 539-542 (Academic Press, 2013).

8 Carter, E. L., Gupta, N. \& Ragsdale, S. W. High Affinity Heme Binding to a Heme Regulatory Motif on the Nuclear Receptor Rev-erb Leads to Its Degradation and Indirectly Regulates Its Interaction with Nuclear Receptor Corepressor. Journal of Biological Chemistry 291, 2196-2222, doi:10.1074/jbc.M115.670281 (2016).

9 Chen, J.-J. \& Zhang, S. Heme-regulated eIF2 $\alpha$ kinase in erythropoiesis and hemoglobinopathies. Blood 134, 1697-1707, doi:10.1182/blood.2019001915 (2019).

10 Fleischhacker, A. S., Carter, E. L. \& Ragsdale, S. W. Redox Regulation of Heme Oxygenase-2 and the Transcription Factor, Rev-Erb, Through Heme Regulatory Motifs. Antioxidants \& Redox Signaling 29, 1841-1857, doi:10.1089/ars.2017.7368 (2017). 
11 Kato, H. et al. Infection perturbs Bach2- and Bach1-dependent erythroid lineage 'choice' to cause anemia. Nature Immunology 19, 1059-1070, doi:10.1038/s41590-018-0202-3 (2018).

12 Motomura, T. et al. Crystal structure and redox properties of a novel cyanobacterial heme protein with a His/Cys heme axial ligation and a Per-Arnt-Sim (PAS)-like domain. Journal of Biological Chemistry 292, 9599-9612, doi:10.1074/jbc.M116.746263 (2017).

13 Nishinaga, M. et al. Heme controls the structural rearrangement of its sensor protein mediating the hemolytic bacterial survival. Communications Biology 4, 467, doi:10.1038/s42003-021-01987-5 (2021).

14 Quick-Cleveland, J. et al. The DGCR8 RNA-Binding Heme Domain Recognizes Primary MicroRNAs by Clamping the Hairpin. Cell Reports 7, 1994-2005, doi:10.1016/j.celrep.2014.05.013 (2014).

15 Shimizu, T., Lengalova, A., Martínek, V. \& Martínková, M. Heme: emergent roles of heme in signal transduction, functional regulation and as catalytic centres. Chemical Society Reviews 48, 5624-5657, doi:10.1039/C9CS00268E (2019).

16 Weitz, S. H. et al. Fe(III) heme sets an activation threshold for processing distinct groups of pri-miRNAs in mammalian cells. bioRxiv, 2020.2002.2018.955294, doi:10.1101/2020.02.18.955294 (2020).

17 Yamawaki, T. et al. Regulatory Switching by Concerted Motions on the Microsecond Time Scale of the Oxygen Sensor Protein FixL. The Journal of Physical Chemistry B 125, 6847-6856, doi:10.1021/acs.jpcb.1c01885 (2021).

18 Yang, J. et al. A Novel Heme-Regulatory Motif Mediates Heme-Dependent Degradation of the Circadian Factor Period 2. Molecular and Cellular Biology 28, 4697-4711, doi:doi:10.1128/MCB.00236-08 (2008).

19 Yi, L., Morgan, J. T. \& Ragsdale, S. W. Identification of a Thiol/Disulfide Redox Switch in the Human BK Channel That Controls Its Affinity for Heme and CO. Journal of Biological Chemistry 285, 20117-20127, doi:10.1074/jbc.M110.116483 (2010).

20 Zenke-Kawasaki, Y. et al. Heme Induces Ubiquitination and Degradation of the Transcription Factor Bach1. Molecular and Cellular Biology 27, 6962-6971, doi:doi:10.1128/MCB.02415-06 (2007).

21 Zheng, H., Williams, J. T., Aleiwi, B., Ellsworth, E. \& Abramovitch, R. B. Inhibiting Mycobacterium tuberculosis DosRST Signaling by Targeting Response Regulator DNA Binding and Sensor Kinase Heme. ACS Chemical Biology 15, 52-62, doi:10.1021/acschembio.8b00849 (2020).

22 Hunter, G. A. \& Ferreira, G. C. Molecular enzymology of 5-Aminolevulinate synthase, the gatekeeper of heme biosynthesis. Biochim. Biophys. Acta 1814, 1467-1473, doi:10.1016/j.bbapap.2010.12.015 (2011).

23 Cao, J. Y. \& Dixon, S. J. Mechanisms of ferroptosis. Cell Mol. Life Sci. 73, 2195-2209 (2016).

24 Malik, Z. \& Djaldetti, M. Cytotoxic effect of hemin and protoporphyrin on chronic lymphocytic leukemia lymphocytes. Exp. Hematol. 8, 867-879 (1980).

25 Tibullo, D. et al. Nuclear Translocation of Heme Oxygenase-1 Confers Resistance to Imatinib in Chronic Myeloid Leukemia Cells. Curr. Pharmac. Design 19, 2765-2770, doi:http://dx.doi.org/10.2174/1381612811319150012 (2013).

26 Levine, A. J. \& Puzio-Kuter, A. M. The control of the metabolic switch in cancers by oncogenes and tumor suppressor genes. Science 330, 1340-1344, doi:10.1126/science.1193494 (2010). 
27 Luengo, A., Gui, D. Y. \& Vander Heiden, M. G. Targeting Metabolism for Cancer Therapy. Cell Chem Biol 24, 1161-1180, doi:10.1016/j.chembiol.2017.08.028 (2017).

28 Martinez-Outschoorn, U. E., Peiris-Pages, M., Pestell, R. G., Sotgia, F. \& Lisanti, M. P. Cancer metabolism: a therapeutic perspective. Nat Rev Clin Oncol 14, 11-31, doi:10.1038/nrclinonc.2016.60 (2017).

29 Steinberg, P. Red Meat-Derived Nitroso Compounds, Lipid Peroxidation Products and Colorectal Cancer. Foods 8, doi:10.3390/foods8070252 (2019).

30 Fiorito, V., Chiabrando, D., Petrillo, S., Bertino, F. \& Tolosano, E. The Multifaceted Role of Heme in Cancer. Frontiers in Oncology 9, doi:10.3389/fonc.2019.01540 (2020).

31 Martin, O. C. B. et al. Targeting Colon Luminal Lipid Peroxidation Limits Colon Carcinogenesis Associated with Red Meat Consumption. Cancer Prev Res (Phila) 11, 569-580, doi:10.1158/1940-6207.Capr-17-0361 (2018).

32 Sohoni, S. et al. Elevated Heme Synthesis and Uptake Underpin Intensified Oxidative Metabolism and Tumorigenic Functions in Non-Small Cell Lung Cancer Cells. Cancer Research 79, 2511-2525, doi:10.1158/0008-5472.Can-18-2156 (2019).

33 Ijssennagger, N. et al. Gut microbiota facilitates dietary heme-induced epithelial hyperproliferation by opening the mucus barrier in colon. Proceedings of the National Academy of Sciences 112, 10038-10043, doi:10.1073/pnas.1507645112 (2015).

34 Fiorito, V. et al. The heme synthesis-export system regulates the tricarboxylic acid cycle flux and oxidative phosphorylation. Cell Reports 35, 109252, doi:https://doi.org/10.1016/j.celrep.2021.109252 (2021).

35 Vander Heiden, M. G., Cantley, L. C. \& Thompson, C. B. Understanding the Warburg effect: the metabolic requirements of cell proliferation. Science 324, 1029-1033, doi:10.1126/science.1160809 (2009).

36 Wise, D. R. \& Thompson, C. B. Glutamine addiction: a new therapeutic target in cancer. Trends Biochem Sci 35, 427-433, doi:10.1016/j.tibs.2010.05.003 (2010).

37 Cluntun, A. A., Lukey, M. J., Cerione, R. A. \& Locasale, J. W. Glutamine Metabolism in Cancer: Understanding the Heterogeneity. Trends Cancer 3, 169-180, doi:10.1016/j.trecan.2017.01.005 (2017).

38 Meyers, R. M. et al. Computational correction of copy number effect improves specificity of CRISPR-Cas9 essentiality screens in cancer cells. Nat Genet 49, 17791784, doi:10.1038/ng.3984 (2017).

39 Nguyen, Tuan A. et al. Functional Anatomy of the Human Microprocessor. Cell 161, 1374-1387, doi:https://doi.org/10.1016/j.cell.2015.05.010 (2015).

40 Fratz, E. J., Stojanovski, B. M. \& Ferreira, G. C. in Handbook of Porphyrin Science, Heme Biochemistry Vol. 26 Heme Biochemistry (eds G. C. Ferreira, K.M. Kadish, K.M. Smith, \& R. Guilard) Ch. 119, 3-68 (World Scientific Publishing Co. Pte. Ltd., 2014).

41 Bishop, D. F., Henderson, A. S. \& Astrin, K. H. Human $\delta$-aminolevulinate synthase: Assignment of the housekeeping gene to $3 \mathrm{p} 21$ and the erythroid-specific gene to the $\mathrm{X}$ chromosome. Genomics 7, 207-214, doi:https://doi.org/10.1016/0888-7543(90)90542-3 (1990).

42 Tsherniak, A. et al. Defining a Cancer Dependency Map. Cell 170, 564-576 e516, doi:10.1016/j.cell.2017.06.010 (2017).

43 Ardlie, K. The Genotype-Tissue Expression (GTEx) pilot analysis: Multitissue 1252 gene regulation in humans. Science, 648-660.

44 Lonsdale, J. et al. The Genotype-Tissue Expression (GTEx) project. Nature Genetics $\mathbf{4 5}$, 580-585, doi:10.1038/ng.2653 (2013). 
45 Chang, K. et al. The Cancer Genome Atlas Pan-Cancer analysis project. Nature Genetics 45, 1113-1120, doi:10.1038/ng.2764 (2013).

46 Dempster, J. M. et al. Agreement between two large pan-cancer CRISPR-Cas9 gene dependency data sets. Nat Commun 10, 5817, doi:10.1038/s41467-019-13805-y (2019).

47 Pacini, C. et al. Integrated cross-study datasets of genetic dependencies in cancer. Nat Commun 12, 1661, doi:10.1038/s41467-021-21898-7 (2021).

48 Zhu, X. G. et al. Functional Genomics In Vivo Reveal Metabolic Dependencies of Pancreatic Cancer Cells. Cell Metab 33, 211-221.e216, doi:10.1016/j.cmet.2020.10.017 (2021).

49 Tang, Z. et al. GEPIA: a web server for cancer and normal gene expression profiling and interactive analyses. Nucleic Acids Research 45, W98-W102, doi:10.1093/nar/gkx247 (2017).

50 Tolosano, E., Fagoonee, S., Morello, N., Vinchi, F. \& Fiorito, V. Heme scavenging and the other facets of hemopexin. Antioxid Redox Signal 12, 305-320, doi:10.1089/ars.2009.2787 (2010).

51 Canesin, G. et al. Scavenging of Labile Heme by Hemopexin Is a Key Checkpoint in Cancer Growth and Metastases. Cell Rep 32, 108181, doi:10.1016/j.celrep.2020.108181 (2020).

52 Elia, I. \& Haigis, M. C. Metabolites and the tumour microenvironment: from cellular mechanisms to systemic metabolism. Nat Metab 3, 21-32, doi:10.1038/s42255-02000317-z (2021).

53 Hooda, J. et al. Enhanced Heme Function and Mitochondrial Respiration Promote the Progression of Lung Cancer Cells. PLOS ONE 8, e63402, doi:10.1371/journal.pone.0063402 (2013).

54 Ma, Y. et al. The relationship between early embryo development and tumourigenesis. $J$ Cell Mol Med 14, 2697-2701, doi:10.1111/j.1582-4934.2010.01191.x (2010).

55 Smith, D. G. \& Sturmey, R. G. Parallels between embryo and cancer cell metabolism. Biochem Soc Trans 41, 664-669, doi:10.1042/BST20120352 (2013).

56 Li, L. et al. Single-Cell RNA-Seq Analysis Maps Development of Human Germline Cells and Gonadal Niche Interactions. Cell Stem Cell 20, 891-892, doi:10.1016/j.stem.2017.05.009 (2017).

57 Yan, L. et al. Single-cell RNA-Seq profiling of human preimplantation embryos and embryonic stem cells. Nat Struct Mol Biol 20, 1131-1139, doi:10.1038/nsmb.2660 (2013).

58 Penzo-Méndez, A., Dy, P., Pallavi, B. \& Lefebvre, V. Generation of mice harboring a Sox4 conditional null allele. Genesis 45, 776-780, doi:10.1002/dvg.20358 (2007).

59 Sasai, K. et al. Targeted disruption of Aurora A causes abnormal mitotic spindle assembly, chromosome misalignment and embryonic lethality. Oncogene 27, 4122-4127, doi:10.1038/onc.2008.47 (2008).

60 Oka, T. et al. Metabolic abnormalities in adult T-cell leukemia/lymphoma and induction of specific leukemic cell death using photodynamic therapy. Scientific Reports 8, 14979, doi:10.1038/s41598-018-33175-7 (2018).

61 Krieg, R. C., Messmann, H., Rauch, J., Seeger, S. \& Knuechel, R. Metabolic characterization of tumor cell-specific protoporphyrin IX accumulation after exposure to 5-aminolevulinic acid in human colonic cells. Photochem Photobiol 76, 518-525, doi:10.1562/0031-8655(2002)076<0518:mcotcs $>2.0 . \operatorname{co} ; 2$ (2002). 
62 Maher, S. P. et al. An adaptable soft-mold embossing process for fabricating opticallyaccessible, microfeature-based culture systems and application toward liver stage antimalarial compound testing. Lab Chip 20, 1124-1139, doi:10.1039/c91c00921c (2020).

63 Egan, E. S. et al. Malaria. A forward genetic screen identifies erythrocyte CD55 as essential for Plasmodium falciparum invasion. Science 348, 711-714, doi:10.1126/science.aaa3526 (2015).

64 Villeneuve, L. Ex vivo photodynamic purging in chronic myelogenous leukaemia and other neoplasias with rhodamine derivatives. Biotechnol Appl Biochem 30, 1-17 (1999).

65 de Lima, M. \& Shpall, E. J. Ex-vivo purging of hematopoietic progenitor cells. Curr Hematol Rep 3, 257-264 (2004).

66 Kobuchi, H. et al. Mitochondrial localization of ABC transporter ABCG2 and its function in 5-aminolevulinic acid-mediated protoporphyrin IX accumulation. PLoS One 7, e50082, doi:10.1371/journal.pone.0050082 (2012).

67 Taketani, S., Inazawa, J., Nakahashi, Y., Abe, T. \& Tokunaga, R. Structure of the human ferrochelatase gene. Exon/intron gene organization and location of the gene to chromosome 18. Eur J Biochem 205, 217-222, doi:10.1111/j.1432-1033.1992.tb16771.x (1992).

68 Whitcombe, D. M. et al. Assignment of the human ferrochelatase gene (FECH) and a locus for protoporphyria to chromosome 18q22. Genomics 11, 1152-1154, doi:https://doi.org/10.1016/0888-7543(91)90044-F (1991).

69 Chenais, B., Andriollo, M., Guiraud, P., Belhoussine, R. \& Jeannesson, P. Oxidative stress involvement in chemically induced differentiation of K562 cells. Free Radic Biol Med 28, 18-27, doi:10.1016/s0891-5849(99)00195-1 (2000).

70 Merryweather-Clarke, A. T. et al. Distinct gene expression program dynamics during erythropoiesis from human induced pluripotent stem cells compared with adult and cord blood progenitors. BMC Genomics 17, 817, doi:10.1186/s12864-016-3134-z (2016).

71 Merryweather-Clarke, A. T. et al. Global gene expression analysis of human erythroid progenitors. Blood 117, e96-108, doi:10.1182/blood-2010-07-290825 (2011).

72 Tirosh, I. et al. Dissecting the multicellular ecosystem of metastatic melanoma by singlecell RNA-seq. Science 352, 189-196, doi:10.1126/science.aad0501 (2016).

$73 \mathrm{Wu}, \mathrm{S}$. Z. et al. Stromal cell diversity associated with immune evasion in human triplenegative breast cancer. The EMBO Journal 39, e104063, doi:https://doi.org/10.15252/embj.2019104063 (2020).

74 Manwani, D. \& Bieker, J. J. The erythroblastic island. Curr Top Dev Biol 82, 23-53, doi:10.1016/S0070-2153(07)00002-6 (2008).

75 Socolovsky, M. Exploring the erythroblastic island. Nat Med 19, 399-401, doi:10.1038/nm.3156 (2013).

76 Lukanidin, E. \& Sleeman, J. P. Building the niche: the role of the S100 proteins in metastatic growth. Semin Cancer Biol 22, 216-225, doi:10.1016/j.semcancer.2012.02.006 (2012).

77 Icard, P. \& Lincet, H. [The cancer tumor: a metabolic parasite?]. Bull Cancer 100, 427433, doi:10.1684/bdc.2013.1742 (2013).

78 Li, X. Q. et al. NAMPT and NAPRT, Key Enzymes in NAD Salvage Synthesis Pathway, Are of Negative Prognostic Value in Colorectal Cancer. Front Oncol 9, 736, doi:10.3389/fonc.2019.00736 (2019).

79 Krammer, B. \& Plaetzer, K. ALA and its clinical impact, from bench to bedside. Photochem Photobiol Sci 7, 283-289, doi:10.1039/b712847a (2008). 
80 Fisher, C. J. et al. ALA-PpIX mediated photodynamic therapy of malignant gliomas augmented by hypothermia. PLoS One 12, e0181654, doi:10.1371/journal.pone.0181654 (2017).

81 Fujishiro, T. et al. 5-Aminolevulinic acid-mediated photodynamic therapy can target human glioma stem-like cells refractory to antineoplastic agents. Photodiagnosis Photodyn Ther 24, 58-68, doi:10.1016/j.pdpdt.2018.07.004 (2018).

82 Shan, L. et al. Increased hemoglobin and heme in MALDI-TOF MS analysis induce ferroptosis and promote degeneration of herniated human nucleus pulposus. Mol Med 27, 103, doi:10.1186/s10020-021-00368-2 (2021).

83 Alexandrova, E. M. et al. Improving survival by exploiting tumour dependence on stabilized mutant p53 for treatment. Nature 523, 352-356, doi:10.1038/nature14430 (2015).

84 Kessel, D. Photodynamic Therapy: A Brief History. J Clin Med 8, 1581, doi:10.3390/jcm8101581 (2019).

85 Hausmann, W. Über die sensibilisierende Wirkung des Hämatoporphyrins. (1913).

86 Toh, T. B., Lim, J. J. \& Chow, E. K. Epigenetics in cancer stem cells. Mol Cancer 16, 29, doi:10.1186/s12943-017-0596-9 (2017).

87 Thakur, C. \& Chen, F. Connections between metabolism and epigenetics in cancers. Semin Cancer Biol 57, 52-58, doi:10.1016/j.semcancer.2019.06.006 (2019).

88 Campbell, S. L. \& Wellen, K. E. Metabolic Signaling to the Nucleus in Cancer. Mol Cell 71, 398-408, doi:10.1016/j.molcel.2018.07.015 (2018).

89 Liao, R. et al. Discovering How Heme Controls Genome Function Through Heme-omics. Cell Rep 31, 107832, doi:10.1016/j.celrep.2020.107832 (2020).

90 Kaasik, K. \& Lee, C. C. Reciprocal regulation of haem biosynthesis and the circadian clock in mammals. Nature 430, 467-471, doi:10.1038/nature02724 (2004).

91 Wang, T. et al. An Analysis of the Multifaceted Roles of Heme in the Pathogenesis of Cancer and Related Diseases. Cancers (Basel) 13, doi:10.3390/cancers13164142 (2021).

92 Mense, S. M. \& Zhang, L. Heme: a versatile signaling molecule controlling the activities of diverse regulators ranging from transcription factors to MAP kinases. Cell Research 16, 681-692, doi:10.1038/sj.cr.7310086 (2006).

93 Finicle, B. T., Jayashankar, V. \& Edinger, A. L. Nutrient scavenging in cancer. Nature Reviews Cancer 18, 619-633, doi:10.1038/s41568-018-0048-x (2018).

94 Bartoschek, M. et al. Spatially and functionally distinct subclasses of breast cancerassociated fibroblasts revealed by single cell RNA sequencing. Nat Commun 9, 5150, doi:10.1038/s41467-018-07582-3 (2018).

95 Sahai, E. et al. A framework for advancing our understanding of cancer-associated fibroblasts. Nat Rev Cancer 20, 174-186, doi:10.1038/s41568-019-0238-1 (2020).

96 Chen, X. \& Song, E. Turning foes to friends: targeting cancer-associated fibroblasts. Nat Rev Drug Discov 18, 99-115, doi:10.1038/s41573-018-0004-1 (2019).

97 Kalluri, R. The biology and function of fibroblasts in cancer. Nat Rev Cancer 16, 582598, doi:10.1038/nrc.2016.73 (2016).

98 Maitra, D. et al. Porphyrin-Induced Protein Oxidation and Aggregation as a Mechanism of Porphyria-Associated Cell Injury. Cell Mol Gastroenterol Hepatol 8, 535-548, doi:10.1016/j.jcmgh.2019.06.006 (2019). 
bioRxiv preprint doi: https://doi.org/10.1101/2022.02.18.481061; this version posted February 19, 2022. The copyright holder for this preprint (which was not certified by peer review) is the author/funder, who has granted bioRxiv a license to display the preprint in perpetuity. It is made available under aCC-BY-NC-ND 4.0 International license. 


\section{Acknowledgments:}

We thank Charles Szerekes for facilitating FACS analysis and the USF genomics program for sharing computing resource and facilities. Also, we thank Rudwan $M$. Soukieh, Saeed Sinan and Thomas D. Nuhfer for discussions and for facilitating work related to the study.

This work was funded by BHC 9BC14, Florida Department of Health, ACS-IRG IRG-14189-19, Women's Health Collaborative. RHYJ also received single-cell genomics protocol development funding from the Gates Foundation OPP1023601, and College of Public Health, University of South Florida.

\section{Author contributions:}

Conceptualization: RHYJ, SRA, GCF, and GAH

Methodology: RHYJ, SRA, GCF, GAH, GWR, NEA, CM, AB, and EMS

Investigation: RHYJ, SRA, GCF, GAH, GWR, and SMS

Visualization: RHYJ

Funding acquisition: RHYJ, GCF, GWR, and SMS

Project administration: RHYJ, GCF, GWR, and SMS

Supervision: RHYJ, GCF, GWR, and SMS

Writing - original draft: RHYJ, GCF, GWR, and SMS

Writing - review \& editing: RHYJ, GCF, GWR, SMS, SRA, and GAH

Competing interests: Authors declare that they have no competing interests.

Data and materials availability: The data used in the study are deposited at $X X X$, and the raw data files are available at XXX.

\section{Supplementary Materials}

Materials and Methods

Figs. $\mathrm{S} 1$ to $\mathrm{S} 8$

Tables S1 to S8 


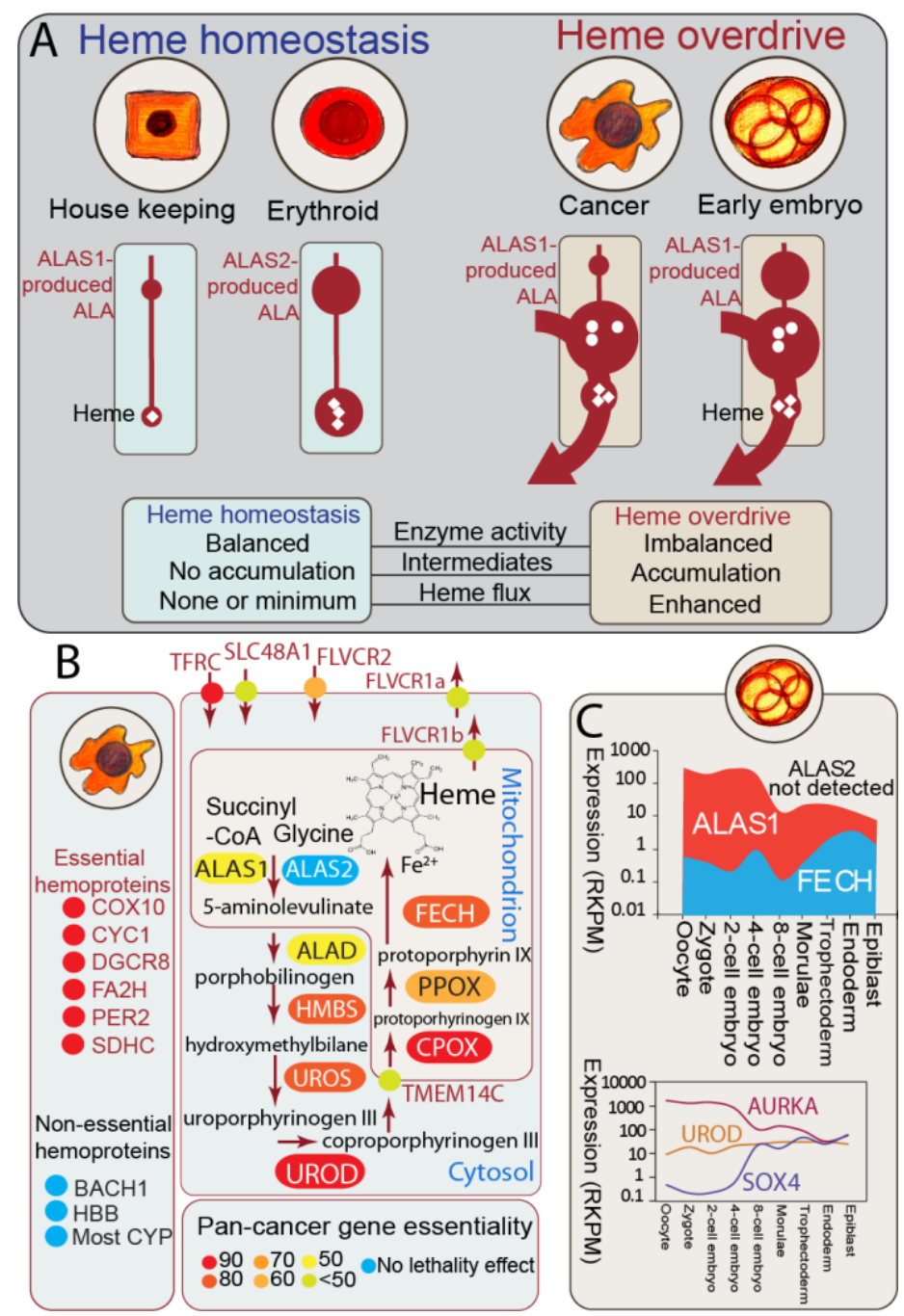

Figure 1. Defining heme overdrive with CRISPR data and early human embryonic stem cell single cell RNAseq. (A) Schematic illustration of heme homeostasis and heme overdrive. Heme homeostasis refers to the heme biosynthetic pathway in normal cells, with the housekeeping enzyme ALAS1 catalyzing the first and rate-limiting step in all cells, with the exception of precursor erythroid cells where the erythroid-specific ALAS isozyme ALAS2 catalyzes the rate-determining step. Heme overdrive refers to the imbalanced (i.e. with aberrantly increased and suppressed enzyme activities) pathway in cancer cells and early human embryonic stem cells, with the ALAS1 isozyme controlling ALA production regardless of the cell type. White circles and white diamonds represent porphyrin intermediates and heme, respectively; Erythroid refers to erythroid precursor cell. ALA, 5aminolevulinate; ALAS, ALA synthase. (B) Defining heme overdrive with CRISPR KO-derived essentiality data in over $\mathbf{3 0 0}$ cancer cell lines. Analysis of CRISPR essentiality data enables mapping of heme overdrive in 27 major types of cancer. Color mapping indicates average cancer cell growth dependence revealed by CRISPR KO of a given gene. UROD has the highest pan-cancer essentiality. (C) Early human embryonic stem cells show features of heme overdrive. The expression levels for $A L A S 1$ and $F E C H$ differ over 100 -fold during the first rounds of embryonic stem cell divisions, but they normalize with the early embryonic development progression. AURKA and SOX4 show expected patterns of expression dynamics during embryogenesis. [Abbreviations: ALA, 5aminolevulinate; ALAS1, housekeeping ALA synthase; ALAS2, erythroid-specific ALA synthase; ALAD, ALA dehydratase (aka porphobilinogen synthase); BACH, transcription regulator BACH (BTB and CNC homology); CPOX, coproporphyrinogen oxidase; COX10, cytochrome $\mathrm{C}$ oxidase assembly homolog 


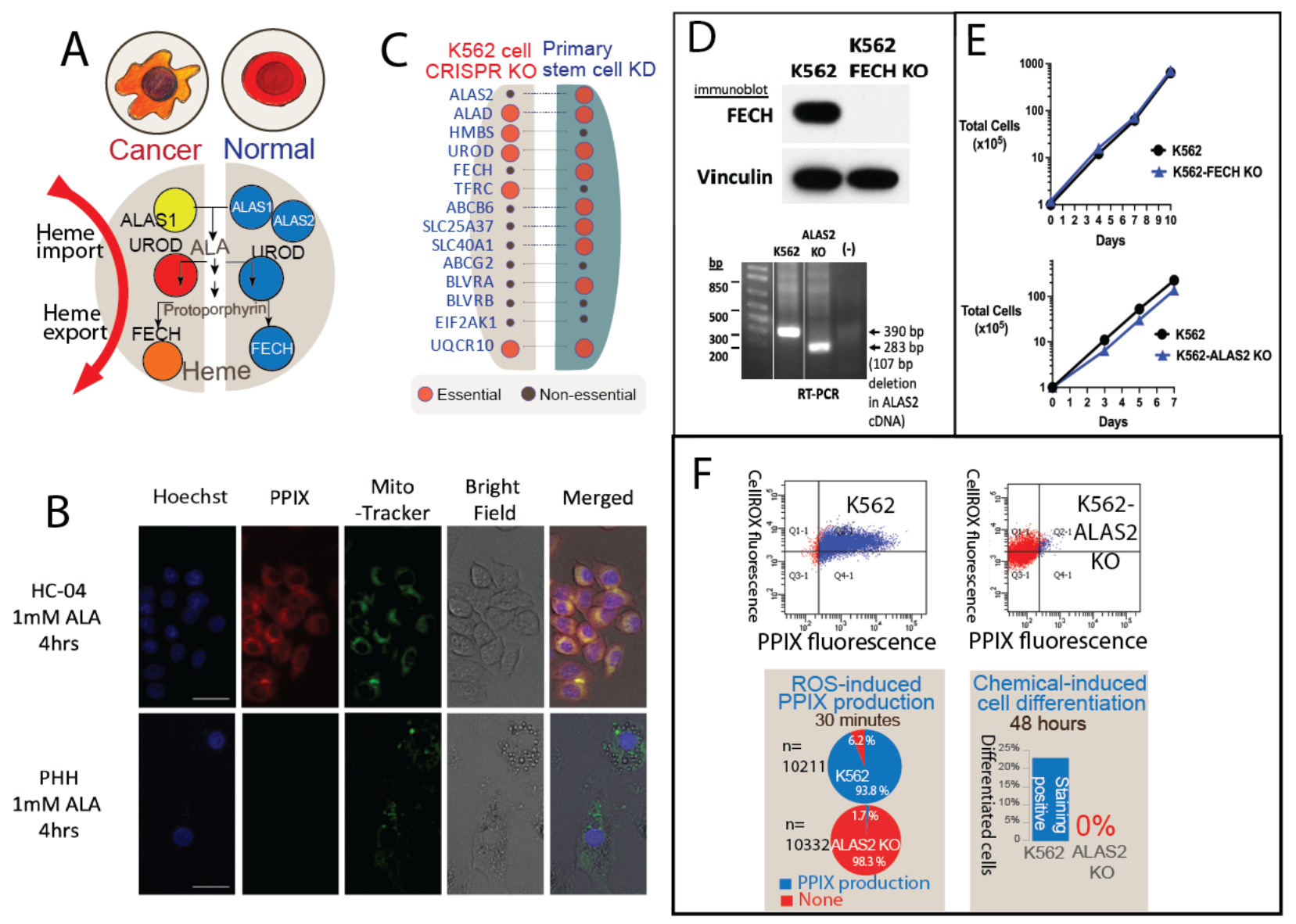

Figure 2. Heme overdrive is unique to cancer cells and absent in normal cells. (A). Schematic illustrations of the key differences of heme metabolism in cancer vs. normal cells. Red, orange and yellow shadings represent cancer gene essentiality in Fig 1B. Blue colors show the normal heme biosynthesis process (B). PPIX autofluorescence (red) is detected in $\mathrm{HC}-04$ liver cancer cells but not in primary human hepatocytes $(\mathrm{PHH})$ following ALA addition, indicating PPIX accumulation in cancer cells but not normal cells. Mitotracker green staining indicates viable cells. Over 1000 cells were assessed for each sample (representative cells shown). PPIX red was not observe in any normal cells. (C). Distinct heme metabolic gene essentiality in stem cell and cancer cells (lethality after loss/deletion of a specific gene). Human primary bone marrow stem cell gene knock down essentiality data from Egan et al (63). Cancer CRISPR KO data are from pan-cancer essentiality analysis (Depmap) (D). Validation of K562-FECH KO cell line generated by CRISPR/Cas9 gene editing. Immunoblots of wild-type K562 and K562-FECH KO whole cell lysates show a complete loss of $\mathrm{FECH}$ protein in $\mathrm{K} 562-\mathrm{FECH} \mathrm{KO}$ cells, with vinculin used as a loading control (top). A $107 \mathrm{bp}$ out of frame CRISPR-induced deletion was identified in the ALAS2 gene (not shown) and due to in inability to identify a definitely specific ALAS2 antibody for immunoblotting, presence of this deletion was confirmed in RT-PCR analysis of K562-ALAS2 KO cells (bottom). (E) Total viable K562 and K562-FECH KO cells (top) and K562 and K562-ALAS2 KO cells (bottom) were determined over time using trypan blue exclusion, and no growth differences were detected. (F). ALAS2 KO K562 cells are arrested in an undifferentiable state. K562 cells readilv differentiated unon ROS induction (tert-hutvl- 

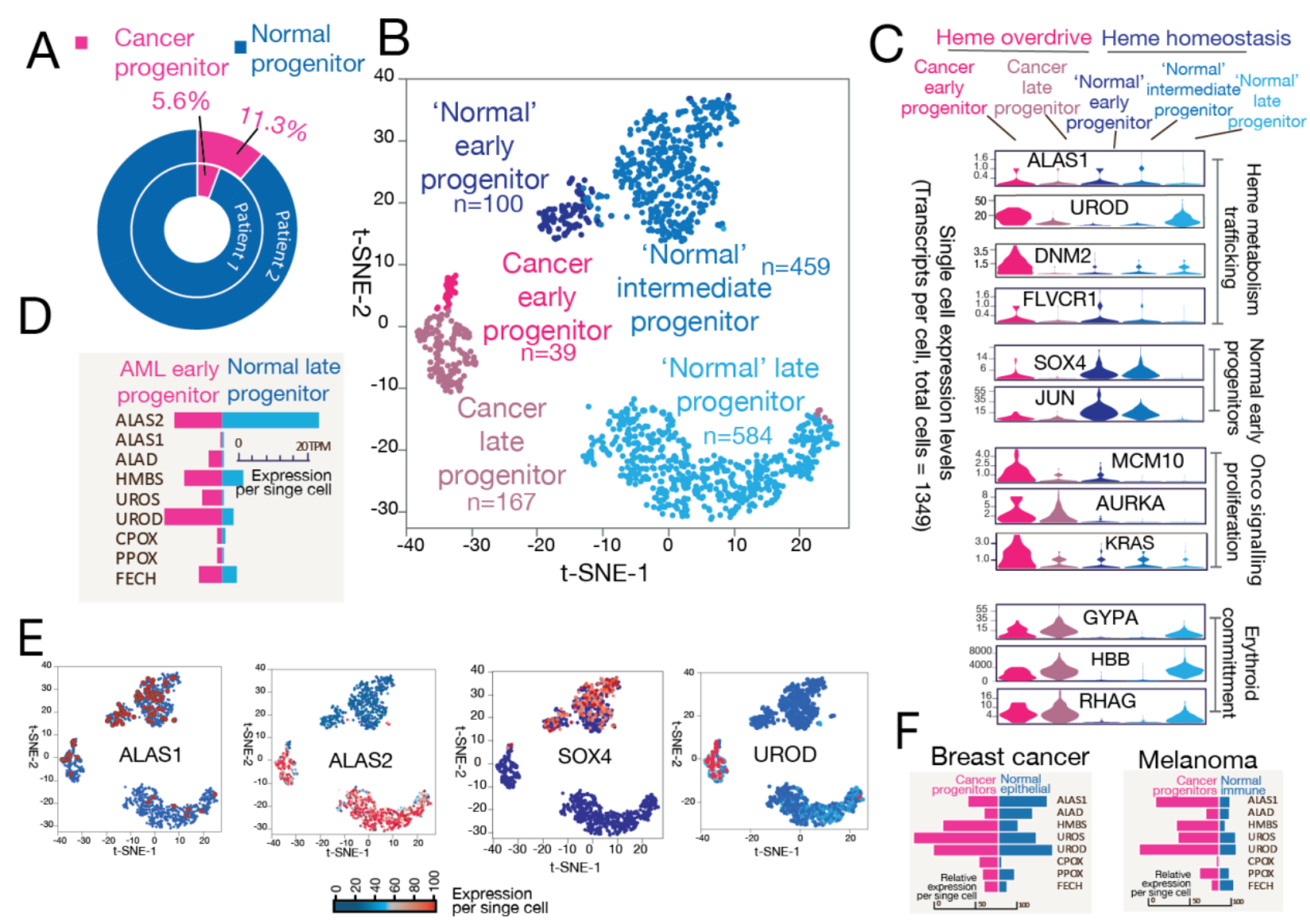

Figure. 3. Single-cell RNA sequencing of human AML bone marrow samples supports heme overdrive as a hallmark of cancer cells. Single-cell transcriptomes were obtained from bone marrow biopsies of AML donors with over $60 \%$ blasts. (A) Single-cell population composition shows only a minor fraction of the cells are cancer progenitors, which are defined by transcriptome embedding and clustering analysis. (B) Identification of distinct single cell populations in the AML patient bone marrow samples by tSNE (t-distributed Stochastic Neighbor Embedding) analysis. A total of 1349 high-quality transcriptomes from patient biopsies were used. (C) Representative genes for heme metabolism, normal early erythroid development, cell proliferation and erythroid commitment process are plotted. (D) UROD, the gene encoding the fifth enzyme of the heme biosynthetic pathway, is highly expressed in cancer early progenitors. HMS and UROS, which encode other intermediate step-catalyzing enzymes, are also over expressed in cancer progenitors. (E) Marker genes delineate cell populations from the patient samples. Note the complementary expression patterns of $A L A S 1$ vs. ALAS2. (F) Solid tumor single cell RNAseq shows overexpression of the genes for the enzymes responsible for the intermediate heme biosynthetic pathway steps in breast cancer and melanoma. 

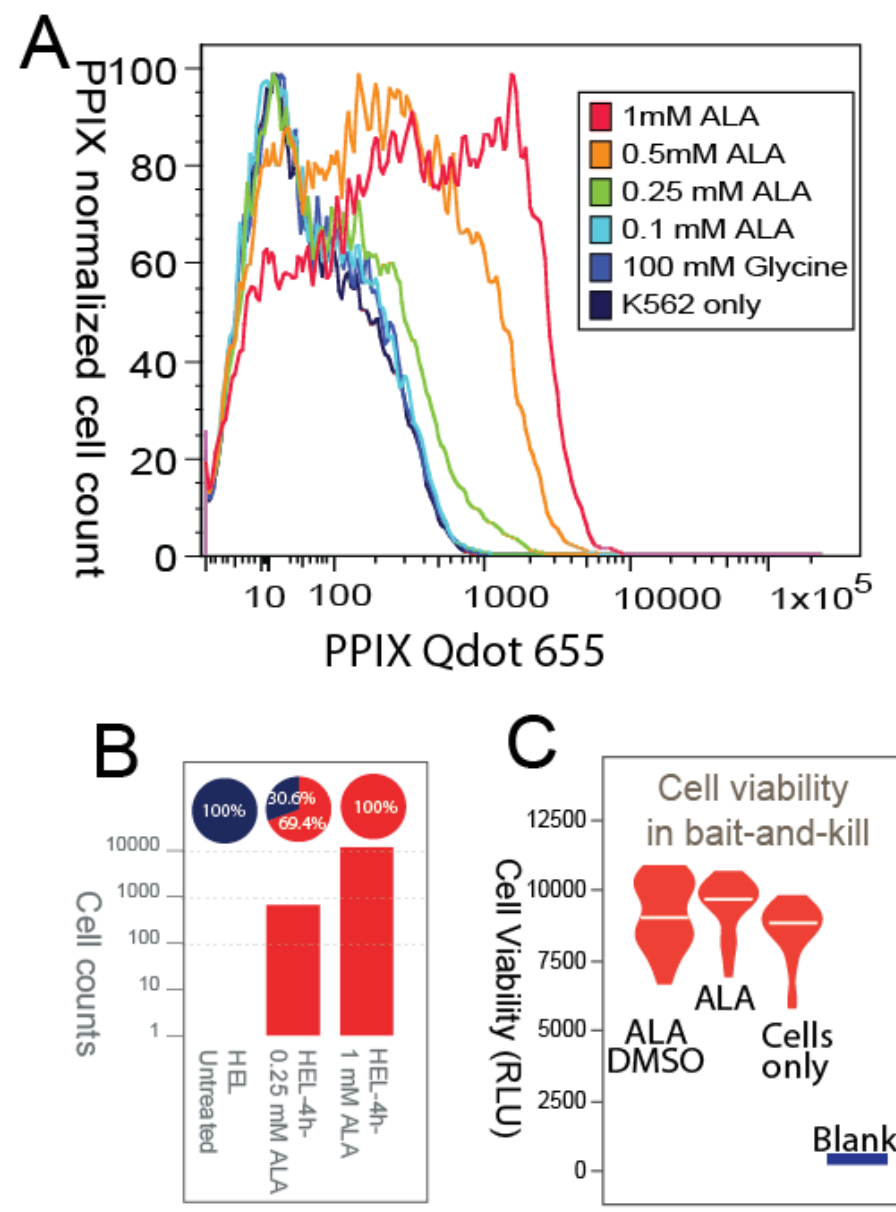
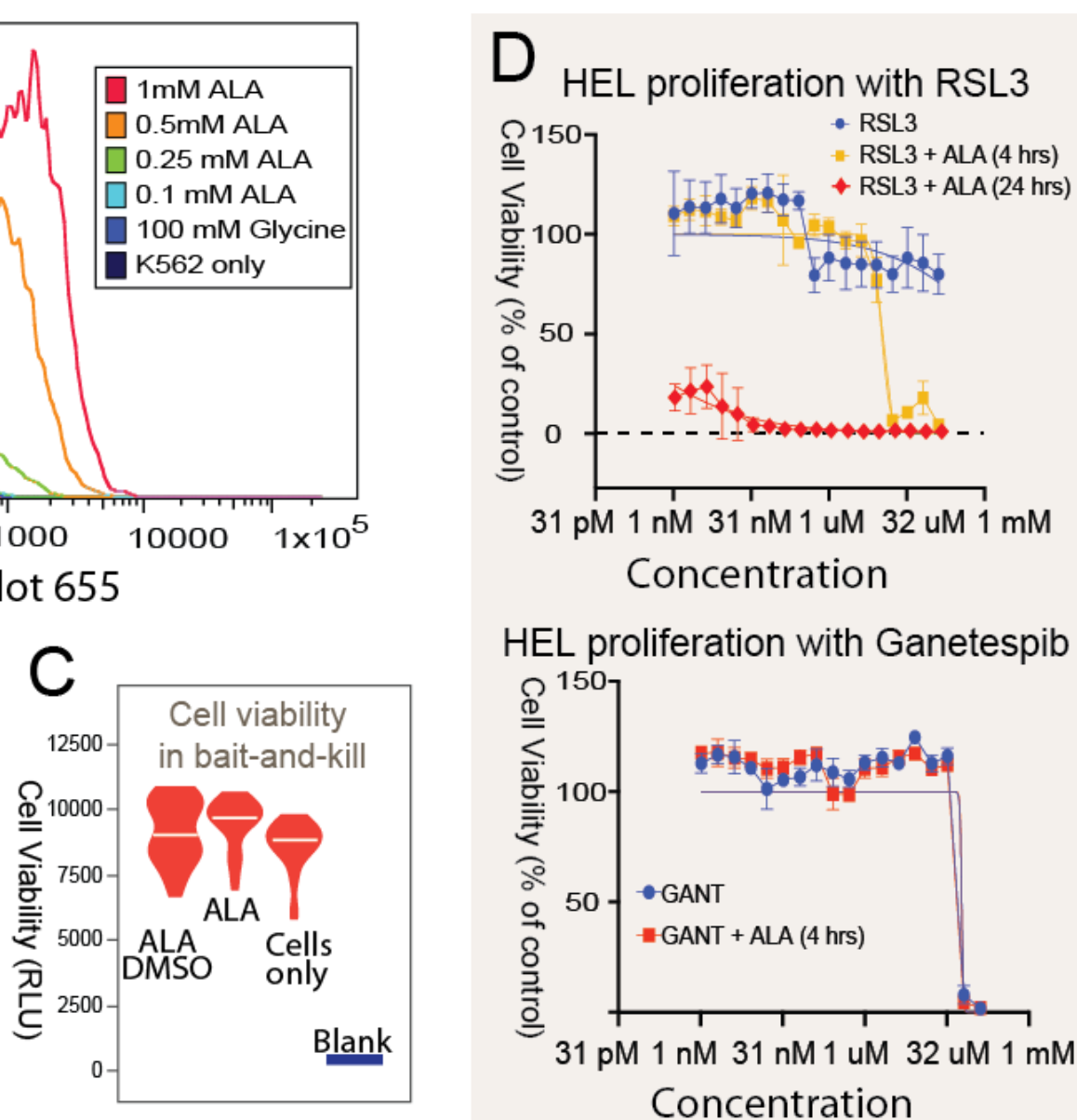

Figure 4: Targeting heme overdrive with a bait-and-kill strategy. (A) The concentration of PPIX accumulated in K562 cells depends on the ALA concentration supplemented to the culture medium. K562 cells were cultured in the absence or presence of $100 \mathrm{mM}$ glycine or ALA $(0.1 \mathrm{mM}, 0.25 \mathrm{mM}, 0.5 \mathrm{~m}$ and $1.0 \mathrm{mM})$ at $37^{\circ} \mathrm{C}$ for $24 \mathrm{~h}$, and PPIX fluorescence was measured by flow cytometry (B) ALA-induced PPIX accumulation in HEL cells. PPIX accumulated in $100 \%$ of the HEL cells ( $\mathrm{n}$ $>10,000$ cells) as detected by PPIX fluorescence 4 hours after treatment with $1.0 \mathrm{mM}$ ALA. Blue shows cells with negative PPIX in flowcytometry; red shows cells with positive PPIX. (C) Cell viability is unaffected by using ALA ("bait") as inducer of PPIX accumulation. HEL cells were treated with ALA $(1 \mathrm{mM})$ at $37^{\circ} \mathrm{C}$ for $24 \mathrm{~h}$, and their viability was calculated from the generated luminescence upon reaction with CellTiterGlo. The assavs were conducted in trinlicate. [RL IJ relative luminescence unitsl. (D) 
bioRxiv preprint doi: https://doi.org/10.1101/2022.02.18.481061; this version posted February 19, 2022. The copyright holder for this preprint (which was not certified by peer review) is the author/funder, who has granted bioRxiv a license to display the preprint in perpetuity. It is made available under aCC-BY-NC-ND 4.0 International license. 